\title{
EFEITO DA TEMPERATURA SOBRE A MASSA ESPECÍFICA DO ÓLEO DE CENOURA
}

\begin{abstract}
César Augusto CANCIAM ${ }^{1}$
Fernanda Schubert Marques dos REIS ${ }^{2}$

Camila Schubert Marques dos REIS ${ }^{3}$

${ }^{1}$ Mestre em Engenharia Química, professor lotado no Departamento Acadêmico de Engenharia Química da Universidade Tecnológica Federal do Paraná - Campus Ponta Grossa, canciam@utfpr.edu.br

${ }^{2}$ Acadêmica do curso de Agronomia na Faculdade Ingá, Uningá, Maringá, fersmr95@hotmail.com

${ }^{3}$ Acadêmica do curso de Medicina Veterinária no Centro Universitário Cesumar, UniCesumar, Maringá, camilasmr97@hotmail.com
\end{abstract}

Recebido em: 15/04/2016 - Aprovado em: 05/08/2016 - Disponibilizado em: 18/12/2016

\begin{abstract}
RESUMO:
A massa específica é uma propriedade termofísica usada na caracterização dos materiais e dependente da temperatura e da pressão. O objetivo deste trabalho foi avaliar o efeito da temperatura sobre a massa específica do óleo de cenoura, entre 313,15 e $373,15 \mathrm{~K}$ e pressão de 1 atm. Utilizando uma equação do tipo Arrhenius e os dados experimentais da massa específica em função da temperatura, o valor encontrado para a energia de ativação foi de $728,8809 \mathrm{~J} . \mathrm{mol}^{-1}$. Na literatura, não foram encontrados valores experimentais da energia de ativação do óleo de cenoura, tornando-se difícil a comparação com o valor encontrado.
\end{abstract}

Palavras-chave: Óleo de cenoura. Efeito. Temperatura. Massa específica.

\section{EFFECT OF TEMPERATURE ON THE DENSITY OF CARROT OIL}

\begin{abstract}
:
The density is a thermophysical property used to characterize the materials and dependent on temperature and pressure. The objective of this work was to evaluate the effect of temperature on the density of carrot oil, between 313.15 and $373.15 \mathrm{~K}$ and pressure of $1 \mathrm{~atm}$. Using a equation of Arrhenius type and the experimental data of density in function of temperature, the value obtained for the activation energy was $728.8809 \mathrm{~J}^{\mathrm{mol}}{ }^{-1}$. In the literature, there is not experimental value for the activation energy of carrot oil, making it difficult to compare with value obtained.
\end{abstract}

Keywords: Carrot oil. Effect. Temperature. Density.

\section{INTRODUÇÃO}

A massa específica, juntamente com a capacidade calorífica, a difusividade térmica, a condutividade térmica e a viscosidade, são consideradas como propriedades termofísicas dos materiais (MAGERROMOV et al., 2008).
O conhecimento do valor dessas propriedades termofísicas é importante em muitas aplicações nas áreas da Ciência e da Engenharia, em particular, nos cálculos relacionados aos balanços energéticos e ao equilíbrio químico (Termodinâmica), na Mecânica dos Fluidos e nas Transferências de 
calor e de massa subjacentes às Operações Unitárias (MATOS, 1998).

A massa específica de um líquido reflete o grau de empacotamento de suas espécies químicas, que é decorrência da intensidade das interações entre elas. Como o volume e a massa específica são grandezas inversamente proporcionais, quanto mais fortes são as interações, mais empacotadas estão as espécies químicas e dessa forma, o volume será menor e, por consequência, a massa específica será maior. Dessa forma, interações mais intensas tendem a se relacionar com massas específicas maiores (BROWN e HOLME, 2009).

Equações do tipo Arrhenius permitem verificar o efeito da temperatura sobre um determinado parâmetro físico-químico, além de permitir sua modelagem matemática (TSEN e KING, 2002; GIAP, 2010).

A Equação (1) corresponde a uma equação do tipo Arrhenius e relaciona a massa específica $(\rho)$ com a temperatura absoluta (T) (TSEN e KING, 2002; CANCIAM, 2014).

$$
\rho=\rho_{\infty} \cdot \exp \left(\frac{E_{a}}{R \cdot T}\right)
$$

Em que $\rho_{\infty}$ corresponde à massa específica quando a temperatura tende ao infinito; $E_{a}$, à energia de ativação e $R$, à constante universal dos gases ideais.
Para se obter os valores de $\rho_{\infty}$ e $E_{a}$ pode ser empregado o método de linearização de curvas proposto por Freund (2004), na qual a Equação (1) é reescrita na forma de:

$$
\ln \rho=\ln \rho_{\infty}+\left(\frac{E_{a}}{R}\right) \cdot \frac{1}{T}
$$

Com relação à energia de ativação $\left(E_{a}\right)$, essa grandeza indica a sensibilidade da massa específica devido à variação da temperatura. Ou seja, para valores elevados da energia de ativação indicam uma mudança mais rápida na massa específica com a temperatura (CANCIAM, 2014).

O óleo de cenoura é rico em carotenoides, apresentando um alto teor de ácidos graxos insaturados. É utilizado principalmente em cosméticos (cremes, loções cremosas, bronzeadores, protetores solares, protetores labiais, máscaras faciais, produtos para massagem, para tratamento de cabelos, de mãos, entre outros), por ser facilmente absorvido pela pele. É considerado emoliente, anti-inflamatório e calmante tópico (ALONSO, 1998).

Ahmed e Abdel-Magied

(2011) comentam da utilização do óleo de cenoura como tônico para nervos e no tratamento de disenterias, vermes, dores uterinas, gota e doenças cardíacas.

O objetivo desse trabalho foi avaliar o efeito da temperatura sobre a massa específica do óleo de cenoura, entre 313,15 e $373,15 \mathrm{~K}$ 
(40 e $100^{\circ} \mathrm{C}$ ) e pressão de 1 atm. Para tanto, foi realizada uma análise de regressão linear de dados experimentais da massa específica do óleo de cenoura em função da temperatura.

\section{MATERIAIS E MÉTODOS}

A Tabela 1 relaciona os dados experimentais da massa específica do óleo de cenoura em função da temperatura. Esses dados foram obtidos do trabalho de Dikko (2015).

Tabela 1. Efeito da temperatura sobre a massa específica do óleo de cenoura

\begin{tabular}{cc}
\hline Temperatura $(\mathrm{K})$ & $\begin{array}{c}\text { Massa específica } \\
\left(\mathrm{kg} \cdot \mathrm{m}^{-3}\right)\end{array}$ \\
\hline 313,15 & 886,77 \\
323,15 & 880,92 \\
333,15 & 874,19 \\
343,15 & 868,08 \\
353,15 & 861,08 \\
363,15 & 854,18 \\
373,15 & 848,12 \\
\hline
\end{tabular}

Fonte: Dikko (2015).

As Equações (3), (4) e (5) fazem parte da análise de regressão linear dos dados emparelhados de $\ln \rho$ e $\frac{1}{T}$, e foram adaptadas do trabalho de Triola (2008).

As Equações (3) e (4) determinam, respectivamente, os valores de $\left(\frac{E_{a}}{R}\right)$ e $\ln \rho_{\infty}$. Enquanto que a Equação (5), o valor do coeficiente de determinação $\left(r^{2}\right)$.

$$
\frac{E_{a}}{R}=\frac{\left\{n \cdot\left[\sum_{i=1}^{n}\left(\frac{1}{T}\right) \cdot \ln \rho\right]-\left[\sum_{i=1}^{n}\left(\frac{1}{T}\right) \cdot \sum_{i=1}^{n} \ln \rho\right]\right\}}{\left\{n \cdot\left[\sum_{i=1}^{n}\left(\frac{1}{T}\right)^{2}\right]-\left[\sum_{i=1}^{n}\left(\frac{1}{T}\right)\right]^{2}\right\}}
$$

$$
\ln \rho_{\infty}=\frac{\left\{\left(\sum_{i=1}^{n} \ln \rho\right)-\left(\frac{E_{a}}{R}\right) \cdot\left(\sum_{i=1}^{n} \frac{1}{T}\right)\right\}}{n}
$$

$$
r^{2}=\frac{\left\{n \cdot \sum_{i=1}^{n}\left\{\left(\frac{1}{T}\right) \cdot(\ln \rho)\right\}-\left\{\left[\sum_{i=1}^{n}\left(\frac{1}{T}\right)\right] \cdot\left[\sum_{i=1}^{n} \ln \rho\right]\right\}\right\}}{\left\{\left\{\left[n \cdot \sum_{i=1}^{n}\left(\frac{1}{T}\right)^{2}\right]-\left[\sum_{i=1}^{n}\left(\frac{1}{T}\right)\right]^{2}\right\}^{1 / 2} \cdot\left\{\left[n \cdot \sum_{i=1}^{n}(\ln \rho)^{2}\right]-\left[\sum_{i=1}^{n} \ln \rho\right]^{2}\right\}^{1 / 2}\right\}}
$$

Com base nos dados indicados na Tabela 1 , os valores de $\left(\frac{E_{a}}{R}\right)$ e $\ln \rho_{\infty}$ foram obtidos a partir das Equações (3) e (4), respectivamente.

\section{Nas Equações (3), (4) e (5), $n$} corresponde ao número de dados emparelhados de $\ln \rho$ em função de $\frac{1}{T}$, que de acordo com a Tabela 1 , equivale a 7.

No cálculo para a determinação do valor da energia de ativação $\left(E_{a}\right)$, considerou-se que o valor da constante universal dos gases ideais $(R)$ equivale a $8,314 \mathrm{~J} \cdot \mathrm{mol}^{-1} \cdot \mathrm{K}^{-1}$ (NETZ e ORTEGA, 2008). 


\section{RESULTADOS E DISCUSSÃO}

A Figura 1 ilustra o gráfico de $\ln \rho$ em função de $\frac{1}{T}$ para o óleo de cenoura.

Figura 1: Gráfico de $\ln \rho$ versus $\frac{1}{T}$ para o óleo de cenoura

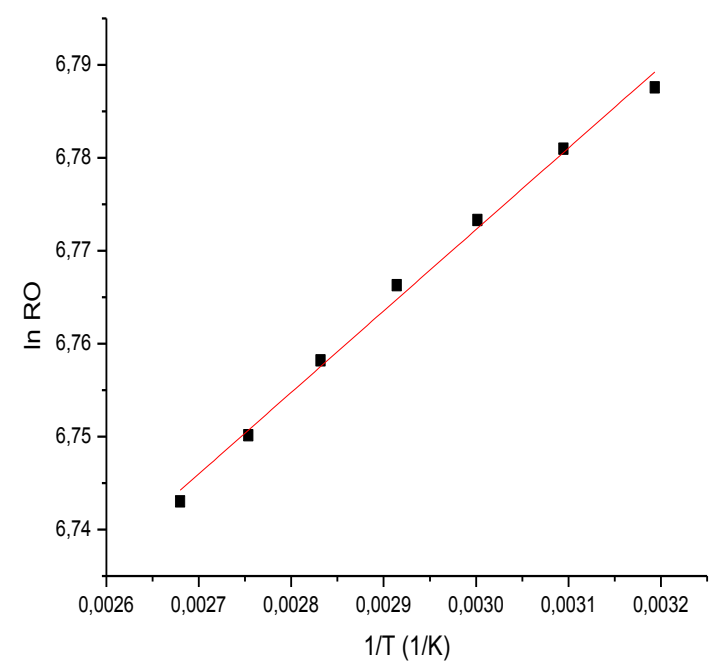

Fonte: Autor.

A Tabela 2 relaciona os resultados obtidos através das Equações (3), (4) e (5).

Tabela 2. Resultados obtidos da análise de regressão linear

\begin{tabular}{cc}
\hline Parâmetros & Valores \\
\hline$\left(\frac{E_{a}}{R}\right)$ & 87,6691
\end{tabular}

Fonte: Autor.

$\ln \rho_{\infty} \quad 6,5093$

Pode-se observar na Tabela 2 que o coeficiente de determinação $\left(r^{2}\right)$ está próximo da unidade.
Com relação ao coeficiente de determinação, Pinheiros e coautores (2009) comentam que esse parâmetro mede a interdependência linear entre as variáveis e avalia a qualidade do ajuste, ou seja, quanto mais próximo o coeficiente de determinação for da unidade, melhor o ajuste da reta em relação aos pontos da dispersão.

Carvalho (2005) comenta que a correlação linear é classificada como muito forte quando os valores do coeficiente de determinação são maiores ou iguais a 0,90 e menores que 1,0. Dessa forma, a correlação linear é classificada como muito forte.

Com base na Tabela 2, o valor obtido da energia de ativação $\left(E_{a}\right)$ equivale a $728,8809 \mathrm{~J}^{\mathrm{mol}}{ }^{-1}$ e $\mathrm{o}$ valor da massa específica quando a temperatura tende ao infinito $\left(\rho_{\infty}\right)$ é de $671,3563 \mathrm{~kg} \cdot \mathrm{m}^{-3}$.

A Tabela 3 fornece os graus de liberdade (GL), a soma dos quadrados médios (SQM) e o quadrado médio (QM) da análise de variância (ANOVA) da análise de regressão linear para o óleo de cenoura.

Tabela 3. Graus de liberdade, soma dos quadrados médios e quadrado médio da ANOVA

\begin{tabular}{lccc}
\hline $\begin{array}{l}\text { Fonte de } \\
\text { variação }\end{array}$ & GL & SQM & QM \\
\hline Regressão & 1 & 0,0016 & 0,0016 \\
Resíduos & 5 & $8,1276 \times 10^{-6}$ & $1,6255 \times 10^{-6}$ \\
Total & 6 & 0,0016 & \\
\hline Fonte: Autor & & &
\end{tabular}

A Tabela 4 indica os valores de $\mathrm{F}$ calculado (Fcal) e o de p. 
Tabela 4. Valores de F calculado e $\mathrm{p}$

\begin{tabular}{ccc}
\hline $\begin{array}{c}\text { Fonte de } \\
\text { variação }\end{array}$ & Fcal & Valor de p \\
\hline $\begin{array}{c}\text { Regressão } \\
\text { Resíduos } \\
\text { Total }\end{array}$ & 968,7628 & $6,4264 \times 10^{-7}$ \\
& & \\
\hline
\end{tabular}

Fonte: Autor.

De acordo com Triola (2008), no modelo de regressão linear simples a função do teste de F é a de testar a significância do efeito de $\mathrm{X}$ sobre $\mathrm{Y}$, ou seja, testar a significância do efeito da temperatura sobre a massa específica. Considerando um nível de significância de 5\%, o valor de Ftab (F tabelado) equivale a 6,61 (TRIOLA, 2008).

Como na Tabela 4 o valor de Fcal é maior que o valor de Ftab, os resultados da ANOVA sugerem que a regressão linear como um todo faz sentido, ou seja, a variável explicativa temperatura influencia globalmente a variável explicada massa específica.

Ara e coautores (2003) comentam que $o$ valor de $p$ varia entre 0 e 1 . Admite-se como um valor crítico de $\mathrm{p}$, valores menores ou iguais a 0,05. Dessa forma, assume-se como margem de segurança $5 \%$ de chance de erro, ou, $95 \%$ de chance de estar certo. Na Tabela 4, pode-se observar que o valor de $\mathrm{p}$ é pequeno, sugerindo que a margem de segurança de erro é pequena.

Buscando na literatura trabalhos associados com a massa específica do óleo de cenoura, constatou-se a ausência desse estudo; o que dificultou as análises dos resultados obtidos nesse trabalho.
Canciam (2014) estudou a influência da temperatura sobre a massa específica de alguns biodieseis metílicos. Considerando os biodieseis metílicos de mamona e algodão (nas temperaturas entre 15 e $90^{\circ} \mathrm{C}$ e pressão de $1 \mathrm{~atm})$, os valores encontrados para a energia de ativação foram de 722,279 e $737,942 \mathrm{~J} . \mathrm{mol}^{-1}$, respectivamente. Dessa forma, pode-se observar que a energia de ativação para o óleo de cenoura $(728,8809$ $\mathrm{J} \mathrm{mol}^{-1}$ ) encontra-se entre os valores encontrados para os biodieseis metílicos de mamona e algodão.

\section{CONCLUSÃO}

O objetivo desse trabalho foi avaliar o efeito da temperatura sobre a massa específica do óleo de cenoura, entre 313,15 e $373,15 \mathrm{~K}$ (40 e $100^{\circ} \mathrm{C}$ ) e pressão de 1 atm.

O valor encontrado para a energia de ativação foi de 728,8809 J.mol ${ }^{-1}$.

Em comparação com outros materiais, constatou-se que a energia de ativação do óleo de cenoura encontra-se entre os valores encontrados para os biodieseis metílicos de mamona $\left(722,279{\mathrm{~J} . \mathrm{mol}^{-1}}^{-1}\right)$ e algodão $(737,942$

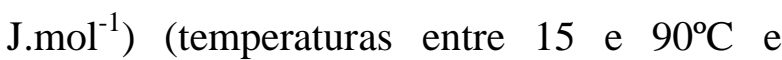
pressão de $1 \mathrm{~atm})$. 


\section{REFERÊNCIAS}

AHMED, A.G.; ABDEL-MAGIED, N. Carrot oil as an antioxidant on some biochemical and haematological disorders in male rats post exposure to $\gamma$ radiation. Journal of Radiation Research and Applied Sciences, v. 4, n. 3(B), p. 957-971, 2011.

ALONSO, J.R.; Tratado de fitomedicina: bases clínicas y farmacológicas. ISIS Ediciones, Buenos Aires, 1998. 1039 p.

ARA, A; MUSETTI, A.B.;

SCHNEIDERMAN, B. Introdução à

Estatística. Editora Edgard Blücher, São

Paulo, 2003. 162p.

BROWN, L.S.; HOLME, T.A. Química geral aplicada à Engenharia. Cengage Learning, São Paulo, 2009. 653p.

CANCIAM, C.A. Influência da temperatura na massa específica de biodieseis metílicos de diferentes origens. Revista de Engenharia e Tecnologia, v. 6, n. 2, p.151-161, 2014.

CARVALHO, M.J.S. Quimiometria e espectroscopia no infravermelho próximo (NIR) e médio (MIR) aplicadas na análise de óleos lubrificantes. Dissertação de Mestrado em Química do Instituto Militar de Engenharia, 2005. 121p.

DIKKO, A.B. Density and surfasse tension relationship of olive oil and carrot oil at diferente temperatures. World Wide Journal of Multidisciplinary Research and

Development, v. 1, n. 2, p. 1-4, 2015.

FREUND, J.E. Estatística aplicada Economia, Administração e Contabilidade. Artmed, Porto Alegre, 2004. 545p.

GIAP, S.G.E. The hidden property of Arrhenius-type relationship: viscosity as a function of temperature. Journal of Physical Science, v.21, n.1, p.29-39, 2010.
MAGERRAMOV, M.A.; ABDULAGATOV, A.I.; AZIZOV, N.D.; ABDULAGATOV, I.M. Pressure- and temperature-dependent density change of juices during concentration. Food Bioprocess Technology, n.1, p.254-269, 2008.

MATOS, M.A.A. Propriedades termofísicas de alguns gases, sólidos e da água. Editora da Universidade de Aveiro, Aveiro, 1998. 30p.

NETZ, P.A.; ORTEGA, G.G. Fundamentos de físico-química: uma abordagem conceitual para as ciências farmacêuticas. Artmed, Porto Alegre, 2008. 299p.

PINHEIROS, J.I.D.; CUNHA, S.B.;

CARVAJAL, S.R.; GOMES, G.C. Estatística básica: a arte de trabalhar com dados. Elsevier, Rio de Janeiro, 2009. 295p.

TRIOLA, M.F. Introdução à Estatística. LTC, Rio de Janeiro, 2008. 720p.

TSEN, J.H.; KING, V.A.E. Density of banana puree as a function of soluble solids concentration and temperature. Journal of Food Engineering, v. 55, n.4, p. 305-308, 2002. 